\title{
Racoon: A Parallel Mesh-Adaptive Framework for Hyperbolic Conservation Laws
}

\author{
J. Dreher and R. Grauer \\ Theoretische Physik I, Ruhr-Universität Bochum, D-44780 Bochum, Germany
}

\begin{abstract}
We report on the development of a computational framework for the parallel, mesh-adaptive solution of systems of hyperbolic conservation laws like the timedependent Euler equations in compressible gas dynamics or Magneto-Hydrodynamics (MHD) and similar models in plasma physics. Local mesh refinement is realized by the recursive bisection of grid blocks along each spatial dimension, implemented numerical schemes include standard finite-differences as well as shock-capturing central schemes, both in connection with Runge-Kutta type integrators. Parallel execution is achieved through a configurable hybrid of POSIX-multi-threading and MPI-distribution with dynamic load balancing. One- two- and three-dimensional test computations for the Euler equations have been carried out and show good parallel scaling behavior. The Racoon framework is currently used to study the formation of singularities in plasmas and fluids.
\end{abstract}

Key words: AMR, mesh refinement, hybrid parallelization, multithread, MPI, load balancing

\section{Introduction}

Adaptive mesh refinement (AMR) techniques have become increasingly popular in recent years for the numerical investigation of dynamic phenomena in fluid dynamics and plasma physics. The underlying idea, as described by Berger and Colella in the context of shock hydrodynamics [1], is to provide a certain spatial resolution of the computational grid that is necessary for the desired accuracy of the solution locally by introducing a hierarchy of subgrids

Email addresses: dreher@tp1.rub.de (J. Dreher), grauer@tp1.rub.de (R. Grauer).

Preprint submitted to Elsevier Science 15 October 2018 
of different sizes and spatial resolutions to cover different regions of the domain. As many problems involve or develop localized small scale structures, it is then possible to compute such critical regions on finer grid blocks while other parts of the domain are well represented on coarser blocks, which can drastically reduce the computational costs compared to using a fine grid in the entire domain. As the system evolves dynamically, with small-scale structures likely to form, move and disappear, their coverage by the recursively refined blocks must be adjusted appropriately in order to ensure proper local resolution. Following this strategy, the computational expenses in terms of memory and CPU usage can easily be reduced to a few percent compared to corresponding simulations based on fixed grids in which the resolution is dictated by the finest structures [12] whenever the phenomena under investigation exhibits strongly localized structures like shocks, near-singularities, boundaryor interface layers and the like.

Successful fluid dynamical and plasma physical applications of this approach today already cover a wide spectrum, including the problem of magnetoconvection in the solar atmosphere [22], the formation of singular current sheets in magneto-hydrodynamics [4],[7] and vortex tubes in hydrodynamics [8], the propagation of solar coronal mass ejections through the heliosphere and their interaction with the Earth's magnetosphere and ionosphere [9], and accretion phenomena in astrophysics [26]. As AMR is merely a supportive technique to improve the economy of computations, a more widespread usage in many different fields can be expected for the future, which, of course, is connected to the progress being made in related areas, e.g. the use of refined physical models, the improvement in numerical methods, and the gain in computational power through modern parallel hardware architectures.

Given these promising perspectives on the one hand and the fact that significant progress occurs in the quite specialized disciplines named above, an obvious challenge is the development of programming environments that combine such modern computational techniques with a degree of flexibility that is necessary to avoid highly specialized, and thus expensive, monolithic solutions in favor of easy adoption, extension and maintenance. Recent steps into this direction are the development of a number of libraries that are specifically targeted at the grid handling tasks in AMR computations like the Fortranbased PARAMESH [16], the freely available DAGH library, written in C++ [2], and the object-oriented designed SAMRAI framework [20]. All of these support parallel execution in distributed memory architectures. Other codes have been developed with more or less specific physical applications in mind, for instance the FLASH code for astrophysical phenomena [3], which is built on top of the PARAMESH library, and the Versatile Advection Code by Tóth [23], which started as a collection of different numerical schemes for fluid and magneto-fluid dynamics, formulated in a dimension-independent fashion, and was recently extended to offer AMR capabilities [12]. This code uses OpenMP 
parallelization and is thereby restricted to (virtually) shared memory systems. The same applies to the incompressible fluid dynamics code used by Grauer et al. for the study of the formation of singular structures [7], [8], in which multi-threading is implemented explicitly through the POSIX standard interface.

In the present paper, we describe Racoon, a framework that has recently been developed to offer an environment for the grid-adaptive solution of conservative systems and related systems. The major motivations here were the possibility to efficiently exploit both shared and distributed memory architectures, and to keep the design as flexible as possible with respect to the numerical schemes used, the physical problems addressed, and forthcoming extensions for more sophisticated simulation techniques. Racoon is implemented in $\mathrm{C}++$ and offers the necessary functionality for computations in 1 to 3 spatial dimensions with the possibility for extensions into higher dimensions.

In the next section, we start with a few general remarks on the overall design philosophy and a brief description of the numerical schemes that have been implemented by now. The grid refinement algorithm and the resulting data communication patterns during the computation are outlined in section 3. Section 4 is devoted to the parallelization strategy, which is a hybrid of multi-threading and interprocess communication through the Message-Passing Interface (MPI), and to some benchmarking results of the scaling in parallel environments. Finally, the conclusion summarizes the key findings and gives a critical assessment of the mixed-mode parallelization.

\section{Design Aspects}

Obviously, an AMR framework, in particular when designed for distributed parallel architectures, exhibits considerably more complexity than conventional computations on fixed grids. Therefore, proper modular code design seems even more advisable than in traditional computations, and the use of corresponding techniques from software engineering that have emerged over the last decades or so, is at least worth being considered. While the adoption of object-oriented design concepts in high performance computing has been somewhat reluctant over the years, there now seems to be a clear tendency to provide at least $\mathrm{C}++$ interfaces to the many standard libraries in this field or even to provide the implementation directly in $\mathrm{C}++$. Reasons for this tendency might be that the run time penalty of $\mathrm{C} / \mathrm{C}++$ codes compared to Fortran codes continues to diminish and, in some cases, has entirely disappeared, in particular when using restricted pointers for de-aliased data access. In fact, some of the AMR frameworks mentioned in the introduction already exploit the potential of object-oriented formulations [20], [2] for the operations 
on grid data and for the data communication between individual grid blocks.

Racoon has been implemented entirely in $\mathrm{C}++$, since this language offers various mechanisms which allow a fine-grained control over the code generation: It supports full runtime polymorphic behavior, but offers also "non-virtual" object-based programming with full compile time resolution which allows function inlining and similar optimizations. In addition, template programming is a technique that combines high abstraction without necessarily sacrificing much runtime performance. Widely used numerical libraries for e.g. linear algebra or FFT are not applicable in the present context as the solution of hyperbolic systems on locally refined meshes with explicit time stepping is a local problem not covered by those algorithms.

In contrast, the numerical schemes employed here, are highly tailored towards conservation laws like compressible gas dynamics or Magneto-Hydrodynamics. They operate on regular numerical meshes and basically involve the computation of numerical fluxes for physical quantities at cell boundaries and an update of the cell integral according to the time integration scheme.

At present, Racoon implements the Kurganov-Tadmor [15] scheme, which is a successor to the one originally proposed by Nessyahu and Tadmor [17], and falls into the class of Lax-Friedrichs type schemes [5]. A major feature here is that they are much simpler to implement than classical Godunov-type schemes [6], [25], [24], as they avoid the spectral decomposition of the conservation equations, and hence are applicable in situations where (approximate) Riemann solvers [19] are unavailable or too expensive. The Kurganov-Tadmor version in particular operates on a non-staggered mesh, making it well-suited for AMR computations. Used in connection with a CWENO-reconstruction, and an appropriate Runge-Kutta integrator, it offers third order accuracy in smooth regions in both space and time. As a cheaper and less powerful alternative, standard second- and fourth-order finite difference discretizations are also implemented.

The entire framework consists of the following major functional components:

- Data management on the grid blocks: Storage, allocation, inter-block communication (e.g., data copy, interpolation), geometry information, block creation and disposal.

- Numerical treatment of a single grid block by means of a given scheme and problem (time stepping, computation of numerical fluxes)

- Definition of the physical system: Variables, flux function (possibly source terms), initial values, boundary values on the physical domain, diagnostics etc.

- Mesh refinement, regridding, load balancing and -distribution.

- Parallelization: Interprocess communication (MPI), thread synchronization 
etc.

- Overall control flow.

Data for physical quantities are kept in standard C-arrays on each individual grid block, and all numerical operations are executed on these arrays with the help of meta-information like array dimensions, number of overlapping ghost points and the like. Comparisons with available libraries like Blitz ++ has shown that they offer no runtime gain compared to the solution chosen here. Every block has a unique identity that specifies the blocks position and size in the domain and keeps direct references (pointers) to its logical neighborhood, i.e. neighbor blocks, the overlayed parent block and its child blocks, as far as they exists. The blocks in turn are organized in a hierarchical manner within a "Grid" object, which basically consists of lookup-tables for the blocks in each refinement level and cached block connectivity information for faster grid traversal.

Numerical schemes are predefined for 1- to 3-dimensional computations and act on a configurable subset of the physical fields. The calculation of the fluxes and sources, boundary values at the physical boundaries etc. occurs through callback functions of appropriate $\mathrm{C}++$ interfaces. For this purpose, a specialized "Problem" instance, that either directly offers the necessary information about the model problem or delegates to other objects, registers itself at program start-up and gets called by the main process control, schemes etc. for the necessary action. Details of the mesh refinement algorithm and the parallelization are given in the following sections.

\section{Mesh refinement}

\subsection{Mesh Refinement Strategy}

In their pioneering paper, Berger and Colella [1] describe a patch-based mesh refinement method for hydrodynamic computations with shocks or highly localized structures: Starting the simulation with a single grid of a given initial resolution, an a-posteriori error estimate is used to identify critical points on the mesh with insufficient spatial resolution. Then, rectangular grid patches of smaller grid spacing and correspondingly higher resolution are introduced to cover those critical points and guarantee the desired accuracy. By applying this method recursively, a hierarchy of grid patches is created so that areas featuring small scale structures are resolved as needed, while the solution is represented and computed on much coarser grids in smooth regions. We call this approach patch-based because the shapes, sizes and positions of the sub-

grids don't follow simple rules and depend on the temporal evolution of the 
system under investigation: Constraints are only their rectangular shape and some nesting conditions which basically state that every grid patch, supplemented with a border region, must lie entirely within an area already covered by patches of the next coarser level. The attractiveness of this approach is the efficient coverage of small scale structures: Once critical points are identified, the grid patches are computed to give an optimal coverage of critical regions.

While this method has been successfully used and implemented in a number of later codes [4], [22], [12], there are some drawbacks: The algorithm to compute the shape and position of the patches under the nesting constraint is far from trivial. Moreover, the fact that the subgrids created this way are irregular in size, shape and position and may abut each other in unpredictable ways means that the regridding procedure for a given refinement level $l$ affects all patches on this level as a whole, making it a non-local task. This, together with the equally irregular data exchange between the subgrids, poses severe difficulties for the parallel execution in distributed memory architectures.

An alternative to this patch-based approach is the regular bisection of grid blocks along each spatial dimension, as used in more recent AMR implementations [16], [18]: Instead of collecting critical points into irregular patches, every grid block that contains at least one critical point is refined as a whole by shadowing or replacing it with a number of equally sized squares (2D) or cubes (3D), which are created by bisecting their "parent" block along every coordinate direction. Using this refinement ratio of 2 , a $d$-dimensional implementation replaces a given block of level $l$ by its $2^{d}$ children of level $l+1$. If the number of cells per block and direction is the same on all grid blocks, the grid resolution increases by a factor of 2 with increasing level $l$. An illustration of this "regular" refinement in 2D is given in Figure 1, and more advanced methods, for example with the option of anisotropic refinement along only one direction or other refinement ratios than 2 , can be found in the literature.

As a consequence of this rather simple approach, a logical tree-hierarchy of grid blocks is created that, despite a less efficient coverage of critical points, has a number of favorable advantages: Well-defined relationships between blocks exist in terms of parent-child relations between refinement levels and neighbor relations on the same level, which makes the implementation of data transfer operations like data copy, interpolation and averaging, relatively easy. Common boundaries shared by neighbor blocks coincide entirely, and a parent's boundaries are contained in the union of its children's boundaries. Due to the tree hierarchy, ordering and traversal of the blocks is straight-forward and block sizes and positions within the domain are well-defined.

This regular subgridding approach was adopted in Racoon because of its compelling simplicity of the regridding procedure, the easy formulation of data communication operations and, in particular, the advantages that the logical 


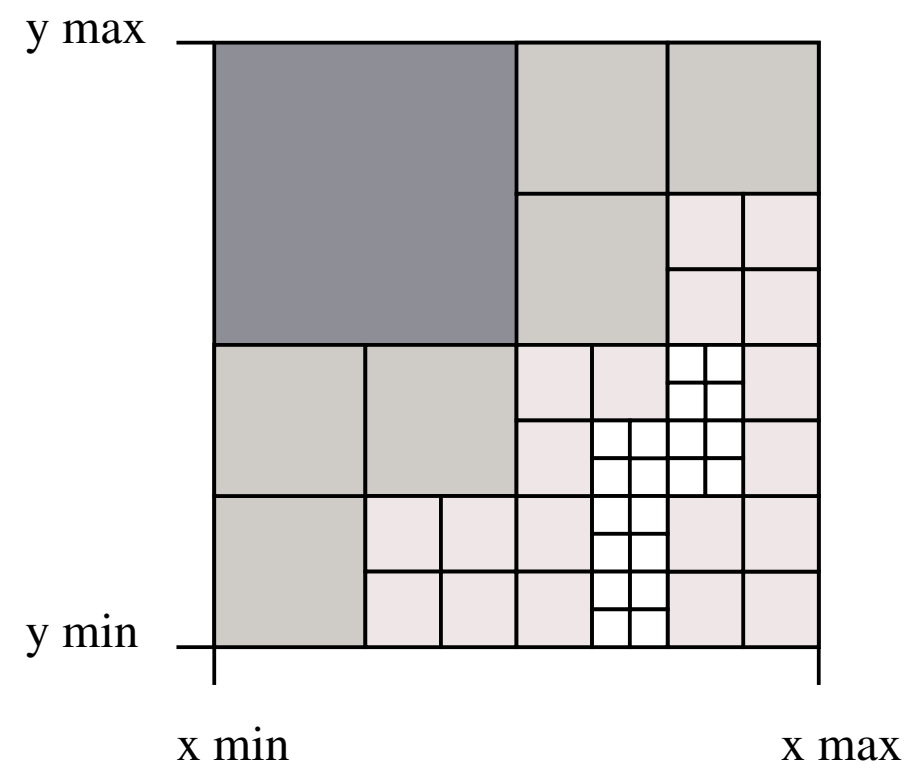

Fig. 1. Illustration of regular grid refinement: The domain is covered by a hierarchy of grid blocks, each of them hosting a fixed number of cells. Upon refinement, a block is shadowed by 4 (in 2D) or 8 (in 3D) equally sized children. Shown here are refinement levels between 1 (upper left) and 4 .

block order offers for the parallel implementation, including the load balancing. In fact, we use a compound block identifier $g=(l, i)$ to assign to every grid block a globally unique identity consisting of the refinement level $l$ and a running index $0 \leq i<2^{l * d}$. With $g$ alone, a block's spatial position and size are determined, as are the corresponding ID's of its neighbors, parent and children if they exists. The mapping between the index $i$ within a given level $l$ and the location of the grid block $(l, i)$ in the domain, i.e. the numbering of blocks, is induced by space-filling curves of the Hilbert type [10], a choice which is made in order to obtain an efficient load distribution strategy for distributed parallel processing as described in section 4.

An apparent penalty compared to the patch-based method is the fact that the coverage of critical sections at first sight seems less efficient with grid block bisecting because the border lines of possible refinement level boundaries are defined a priory. However, this turns out to be less severe than expected if the computations are started with a refinement level $l>0$ right away, i.e. with a domain decomposition: Then, only few further refinements are needed to localize the high resolution regions around critical structures. In addition, a certain margin of increased resolution around critical structures is necessary to accommodate their possible motion relative to the grid between regridding phases. Typical runs start with $l=3$ or $l=4$, corresponding to a domain decomposition into 64 or 256 blocks in 2D and 512 or 4096 in 3D.

An important positive side effect of starting the simulation with a domaindecomposed setup right from the beginning is the gain in execution speed: 
Even in sequential mode, the decomposed domain with relatively small grid blocks (typically $8^{d}$ to $16^{d}$ cells) leads to a much better CPU cache utilization than one large grid block, and this cache efficiency easily comes with a gain of factor 2 or more in execution speed on the tested platforms (i686, Opteron, power4) despite the necessary boundary data copy. In parallel mode, the coarsest grid level is easily parallelized.

A regridding procedure is initiated after a configurable number of time steps by checking every point in every block for a given refinement criterion. The criterion itself is defined together with the physical problem and is typically based on the gradient of some variables as to identify small scale structures in the domain. A more sophisticated and possibly less biased approach might be a thorough error estimate, for instance using Richardson-extrapolation as proposed by Berger and Colella [1]. Grid cells which are found to be critical cause the containing blocks to be flagged for refinement. In addition, those critical points which are close to the block's boundary are used to flag the abutting neighbor block(s) as well in order to communicate the information about critical structures across block boundaries and ensure a buffer zone of high resolution around critical points. Finally, additional refinement marks are set in order to ensure a grid consistency criterion which states that abutting blocks may differ by at most one refinement level. This criterion greatly simplifies the interpolation operation at refinement boundaries and comes at almost no cost.

After this marking step, the entire grid is updated: Flagged blocks, unless already refined, are supplemented by their children, using the actual parent block's data for initialization, while unnecessary refinements are removed by deleting the corresponding child blocks after their actual data was transfered to the coarser parent block. The parent blocks, even if refined, are still kept in the computation. Depending on the integration and time stepping mode that was selected, they are either used to obtain a first approximation during every integration step and provide intermediate boundary values for children and neighbors, or they are simply deactivated and kept passively in the process. Overall bookkeeping of the blocks is handled by "grid" objects, as already mentioned, where the block ID's (level-key pair) are used as keys and iterators are provided for the traversal of the entire grid or blocks of a certain refinement level.

\subsection{Time stepping}

Blocks are integrated quasi-autonomously, which requires bands of ghost cells around the actual physical area that have to be updated after every integration step: For neighboring blocks of the same refinement level, this is just a copy 
of the overlap region like in a domain decomposition, and blocks which abut the physical domain boundary are treated according to the corresponding physical boundary condition. At refinement boundaries, finer blocks receive interpolated ghost cell values from their respective coarser neighbors, while coarser blocks receive averaged values from the fine region.

In order to fulfill the Courant-Friedrichs-Levy (CFL) condition for numerical stability, finer grid blocks will in general need a smaller integration time step than their coarser counterparts. In most applications, this stability restriction is more relevant than the accuracy condition due to the explicit solvers used.

There are two basic ways for the time stepping on locally refined grids: 1 . One common step is used for all grid blocks, dictated by the most stringent conditions found in the entire domain, or 2. different refinement levels use individual time steps so that coarser blocks in general will be integrated with fewer larger steps than fine blocks, which leads to a temporal refinement in addition to the spatial one. The main reason for using the first method is it's simplicity and, for more advanced systems like incompressible flows, the compatibility with additional correction steps (e.g. velocity projection after solving a pressure equation). The advantage of the second approach is, apart from moderate savings in computing time, the fact that also on coarse blocks, the CFL number can stay close to 1 which is favorable for the phase error confinement in some numerical schemes.

One of either methods can be selected in the Racoon time stepping module, and the time step condition itself is always provided by the specific problem instance which gets periodically queried by the time step classes which in turn calculate the individual time steps for each refinement level and the resulting integer factors between them.

When individual time steps $\Delta t_{l}$ are chosen for each level $l$, one step on the entire grid is formulated as a level-wise, recursive procedure (intra-level communication with data copy is left out for clarity):

- step grid blocks on level l, individual $\Delta t_{l}$ :

- save data at refinement boundaries to level $l+1$ (old)

- integrate level $l$ blocks once with time step $\Delta t_{l}$

- if not coarsest level: update boundary values at boundary to coarser blocks as temporal interpolation

- pass spatial interpolation of old an new data at boundaries to level $l+1$ to corresponding level- $l+1$ blocks

- call step grid blocks of level $l+1 \Delta t_{l} / \Delta t_{l+1}$ times

- if last step on level $l$, pass latest coarsened volume data to level $l$-1-blocks at refinement boundaries

This methods requires that shadowed coarse grid blocks at boundaries to finer 
blocks are included in the integration in order to provide temporal interpolations of boundary data for the abutting finer blocks. It is evident that the algorithm results in a level-by-level integration with intermediate data transfer between levels.

If one common time step is selected, the procedure is considerably simpler:

- step all grid blocks with common $\Delta t$ :

- step all blocks once with time step $\Delta t$

- pass coarsened boundary data from fine to coarse levels at refinement boundaries, starting with finest level

- pass interpolated boundary data from coarse to fine levels at refinement boundaries, starting with coarsest level

Even here, the boundary data exchange has to occur separate for the fine-tocoarse direction and vice-versa due to the implicit data dependency: For the calculation of the (new) local interpolation to be passed to the fine ghost cells, the coarse blocks must have new values in their own boundary (ghost) cells already available.

While it would be possible in principle, to keep track of the data flow for each individual block and to use the data dependency as a criterion to start integrating a block, we have followed the approach of a level-by-level global synchronization. This facilitates the entire flow control and seems by far the most practical way to go as the dependency graphs between blocks get arbitrarily entangled after local refinement (note that data is exchanged not only between face neighbors, but, depending on the numerical scheme, also diagonal neighbors; interpolation depends on previous intra-level exchange etc.). In particular in the case of multi-threaded execution to be described in the next section, this data tracking would require additional thread-synchronization for consistent housekeeping, and we expect that the overall gain would be small compared to the necessary effort. The global synchronization doesn't require a separate global communication action: It's simply provided by synchronizing threads within each process after all external communication has been finished.

\section{Parallelization}

High performance computing today requires efficient parallel execution. Adaptive mesh refinement suggests the fundamental parallelization strategy of handling every single grid block as a quasi independent piece of work for each integration step, and assign the blocks to CPUs for integration. The existence of ghost cells around the blocks, which effectively represent a buffer 
zone, allows this approach to be implemented directly. However, it must be kept in mind that AMR execution, and thereby its parallelization, is considerably more complex than a pure domain decomposition: Blocks are recursively advanced level by level as described in chapter 3, and information like interpolated boundary values and flux corrections are exchanged between parents and children at level changes. In shared memory architectures, the corresponding communication paths are still workable without particular consideration: For instance, Keppens and Tóth [13] report run time speed-up factors of up to 4.9 on a 16 CPU SGI Origin using manual parallelization with OpenMP. These results are in agreement with the scaling that Grauer et al. [8] obtained using multi-threading.

However, as the trend in high performance computing moves towards cluster architectures, distributed parallelism must be addressed in order to exploit these platforms. The approach of regular subgridding as presented in chapter 3 is mainly motivated to ease distributed parallelism: An obvious advantage here is that the regular interfaces between neighboring (and nested) blocks facilitate the programming, making a distributed implementation through the Message Passing Interface (MPI) workable. Moreover, equal block sizes mean roughly the same computational load per block, which greatly facilitates the dynamical load balancing. A further aspect is the fact that we can globally identify a grid block by means of its block ID, consisting of the refinement level and a running index, across address spaces, which makes it easy to send block-to-block messages between computing nodes. Finally, the indexing of blocks of a given level by means of Hilbert curves, as already mentioned in chapter 3, offers a distribution strategy that aims at minimizing the inter-node communication.

With Racoon, an attempt has been made to combine multi-threading with MPI distribution in order to adopt to cluster architectures consisting of SMP nodes. In the following subsections, this approach will be described in detail together with benchmark results of the parallel performance.

\subsection{Hilbert Curve Ordering of Blocks and Load Distribution}

As already mentioned, Hilbert type space filling curves are used to order and distribute the individual grid blocks in a way which aims at minimizing the communication between concurrent threads of program execution and between processes. Hilbert space filling curves of level $l$ in $d$-dimensional space provide a mapping between the interval $[0,1]$ and the domain $[0,1]^{d}$ with the property that neighborhood or proximity tends to be conserved under this mapping. This means that points being close to each other in the interval $[0,1]$ tend to be mapped to points in space which are also have a small distance in $R^{d}$ and 

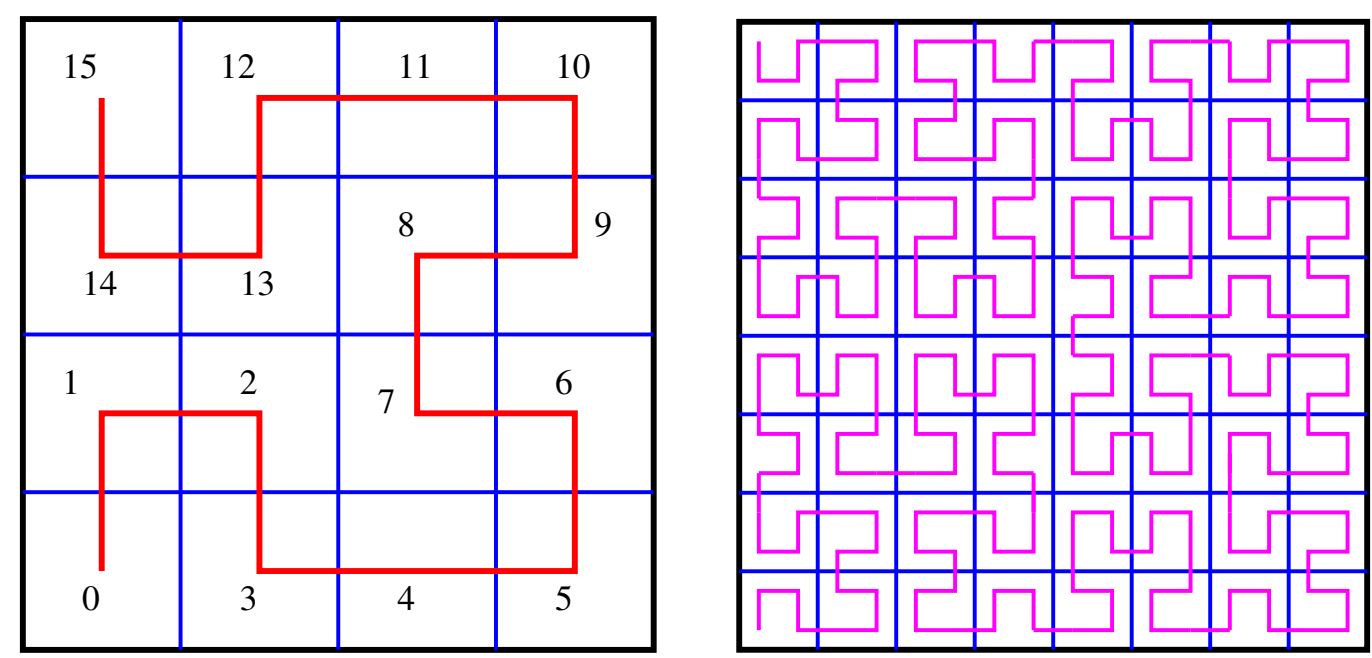

Fig. 2. Two-dimensional Hilbert curves of level 2 (left) and 3 (right), respectively. Each of the squares corresponds to a potential grid block. Blocks of a given level are ordered according to their position on the visiting Hilbert curve in order to achieve clustering on computing nodes.

vice versa, a feature which is utilized in several areas of computer science. For illustration, the 2D Hilbert curves of level 1 and level 2 are shown in Figure 2. This tendency of neighborhood conservation is used to determine the distribution of blocks among separate computing nodes (processes/threads) in a way that tends to assign neighboring blocks to the same node and thereby minimize inter node communication. A similar approach is used in the PARAMESH library by MacNeice et al. [16] and theoretical investigations into the efficiency of Hilbert curves in load distribution are given in e.g. [29] and [30].

To achieve this communication-efficient distribution, blocks of a given level $l$ are ordered according to their Hilbert index $i$, and this sequence is divided into equally sized partitions (up to rounding error) for computing nodes. With this mechanism, every block is assigned to a node, and physically close regions of the domain tend to end up on the same node. It should be noted that it is also possible to formulate a order not only per level but of all blocks, which additionally conserves the proximity between parents and children. This, however turned out to be disadvantageous for our application: While computational load balance can be achieved by weighting every block with a cost factor according to its refinement level (and thereby to the number of integration steps), the fact that only blocks of a certain level are integrated at a given time causes idle periods on some nodes unless every node has the same number of blocks for every level.

Figure 3 shows a close-up view of a local grid structure created during a simulation run together with their ordering as induced by the space filling curves. The effect of cluster formation of blocks belonging to the same refinement level (i.e. having the same size) is obvious, and remote communication 


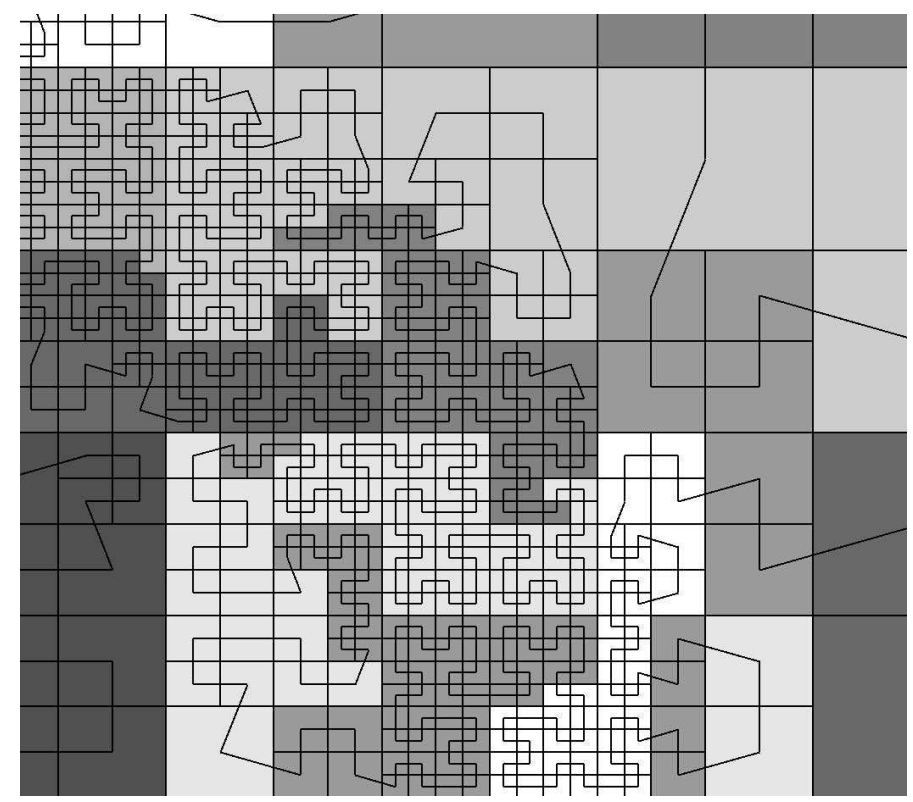

Fig. 3. Close-up view of a number of blocks up to refinement level 6 , taken from an actual simulation run. The gray scale indicates the computer node on which a block is located. Each block consists of $16 \times 16$ cells in this case. Distribution is based on the Hilbert curve ordering on a per-level basis, which leads to the obvious clustering of neighbouring blocks on each level.

between computing nodes is then restricted to the lines dividing these cluster.

In our implementation, load distribution is directly coupled to the grid refinement: After a regridding procedure, which might create or remove blocks on the various nodes, the new load per node is calculated for every level. If the load imbalance exceeds a given threshold, blocks are migrated between the nodes to arrive at the optimal load distribution. Note that the distributed regridding already involves a collective communication, because the refinement criterion applied to a block on one node might flag a neighboring block, possible residing on a different node, as described in section 3. Therefore, grid refinement flags have to be exchanged between nodes before the actual refinement takes place, which then is a local process. Afterwards, the re-distribution is carried out, if necessary.

\subsection{POSIX-Multi-thread Parallelization}

On SMP machines, the use of multi-threading instead of distribution into different processes seems a natural way for parallelization. While OpenMP is an established standard here, Racoon uses explicit thread programming through the standard POSIX interface in order to preserve finer control over the workload distribution and the affinity between CPUs and memory. As mentioned earlier, a general observation is that the efficient cache utilization is crucial for 


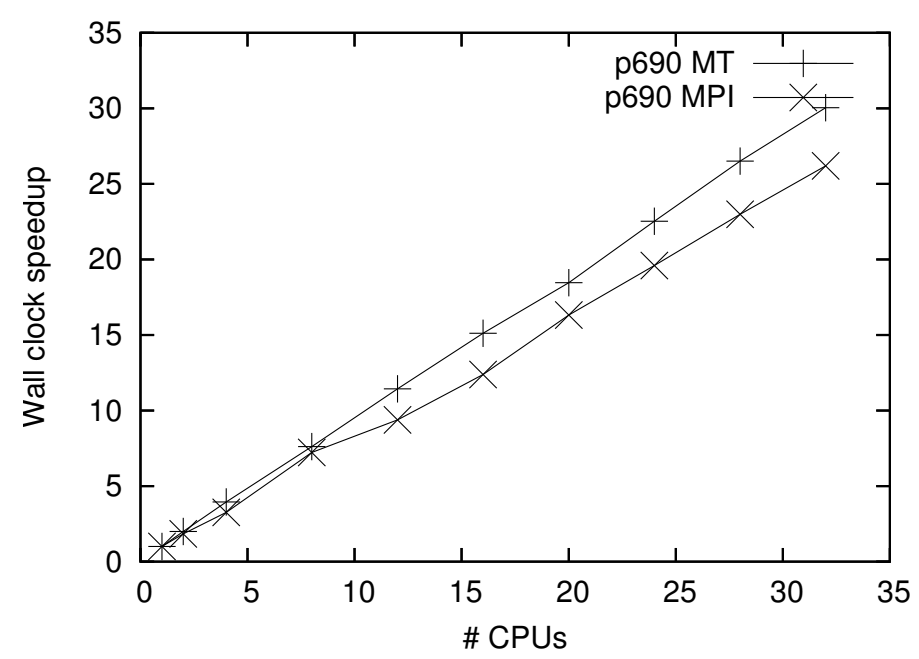

Fig. 4. Wall-clock speed-up of the test application on IBM p690 with multithread parallelization (MT) and single-thread MPI distributed parallelization (MPI), compared to the serial run (at \# CPU=1). The serial execution time is 2253 seconds, floating point rate is $\approx 600$ MFlips. Times exclude data initialization, I/O, regridding.

high computing performance on modern CPU architectures . Therefore, the load distribution and balancing by means of Hilbert curves has been carried over to the multi-thread parallelization in that each POSIX thread (one per $\mathrm{CPU}$ ) operates on a fixed subset of all grid blocks according to the calculated distribution. Moreover, each thread has its own copy of the entire grid (i.e. the block tables and connectivity information) with the peculiarity that only those blocks that are local to the thread are populated with data. Unpopulated blocks act as proxies and contain pointers to the actual data in the "spheres" of other threads if they're in the same address space. All data allocation takes place from within the thread that later operates on the block data in order to ensure that a good CPU-to-memory affinity is achieved also on architectures with non-uniform memory access (NUMA).

With this strict data separation between the threads, not only the numerical operations on the block data, but also the iterations related to grid traversal and retrieval of connectivity information from block objects operate on local copies for each thread. Thus, the fundamental idea is to effectively create thread-private data spaces and basically restrict all memory accesses that might trigger cache-coherency controllers to enforce re-caching operations to the inevitable exchange of boundary data between grid blocks. As mentioned, this exchange is limited by means of the partitioning according to space-filling curves. A typical time step on the grid (or on one grid level, depending on the integration mode) consists of first advancing all blocks (in which the threadallocated data is accessed "privately" by the working thread), followed by data copy which involves overlapping memory access and might cause cache conflicts. 


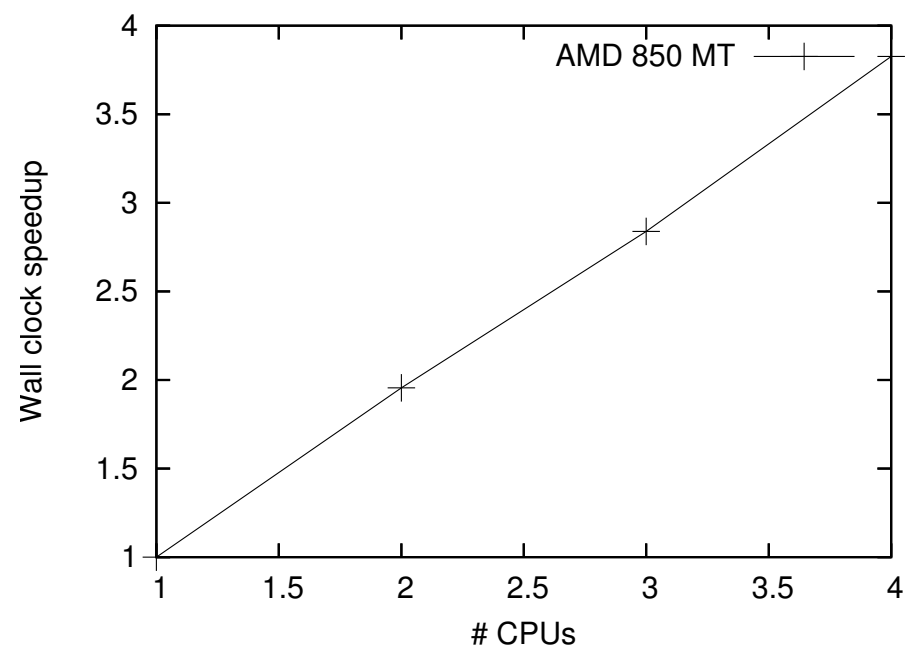

Fig. 5. Wall-clock speed-up of the test application on AMD opteron 850 quad with multithread parallelization (MT) compared to the serial run (\# CPUs = 1). Here, the serial execution time (w/o initialization, I/O, regridding) is 176 seconds. The single thread MPI measurements gave essentially the same results.

We have tested this approach on the JUMP multiprocessor at Forschungszentrum Jülich, which consists of IBM p690 machines, each with 32 Power4+ CPUs at $1.7 \mathrm{GHz}$ clock rate running AIX, and on a local quad AMD Opteron 850 machine with $2.4 \mathrm{GHz}$ CPU clock running Linux with a 2.6.10 kernel. Fig. 4 and 5 show the execution time of test runs with a typical setup. Here, the 3 dimensional Euler equations from gas dynamics used to simulate gas blow-out from dwarf galaxies as a result of supernovae explosions, are integrated for 200 time steps. The numerical scheme is the Kurganov-Tadmor scheme with 3rd order CWENO reconstruction and 2nd order Runge-Kutta integration on grid blocks with $8^{3}$ cells and 2 layers of ghost cells, the number of grid blocks was approximately 4800 on the p690 and 590 on the Opteron 850 quad setting. The execution times given cover only the numerical integration itself, without diagnostics, I/O, regridding or initialization of block data. On the IBM p690 power4+, the measured sustained floating point rate was around 600 MFlips per CPU, which is close to $9 \%$ of the theoretical peak performance and can be considered a reasonable value for this kind of application. The execution time for the communication alone (boundary data exchange by copy, interpolation, averaging) amounts to about $10 \%$ of the total execution time in all cases.

In all cases, the scaling behavior is good to excellent, which, however, was obtained only after further manual optimization: In order to guarantee a tight task affinity between the executing threads and the CPUs, and thereby take full advantage of the affinity between threads and data as described above, we had to explicitly bind the created threads to single CPUs in the machines. In AIX, this was achieved with the "bindprocessor" system function, on Linux with "sched_setaffinity." This manual tuning was essential for the displayed scaling behavior: Without the enforced CPU affinity, the parallel performance 
degraded by about $10--15 \%$ on both platforms, with considerable fluctuations between runs. Binding the threads to CPUs, however, resulted in the well reproducible scaling figures shown in Figures 4 and 5. The interpretation here is that (unwanted) spontaneous task migrations of the computing threads between CPUs during the run leads to random enhancements of the distances to the memory where the corresponding data is allocated and to necessary re-cache operations.

\subsection{MPI Parallelization}

The parallelization over address spaces, necessary for network-based clusters, is similar in principle to the method described above: Here, every process hosts a subset of all grid blocks, but the data exchange now involves message passing with MPI rather than direct memory copies. An explicit serialization of message data has been implemented instead of using the many specialized transfer functions and derived data types of MPI. The reason is again that many different kinds of messages are exchanged with different parts of a ghost cell layer, and their flow is hardly predictable, in particular for the exchange at refinement boundaries. It seems much more straight-forward to compose messages that contain meta information about the message type, the target block and the target region within the block (e.g. which ghost cells) together with the core data itself.

Two different approaches have been followed concerning the message size, number and send time:

1. Each data portion to be transferred (e.g. part of a ghost cell layer of one specific block to one specific neighbor block) is composed into a MPI message and posted asynchronously with MPI_Isend as soon as the sending block is updated. The matching receive requests are posted on the receiving side before the level update, and message completion and dispatch occurs after the level update.

2. Small inter-block messages to the same target process are collected into larger MPI messages of fixed sizes. These are transferred after a level update is finished and dispatched on the basis of the embedded meta information. The need for continuation messages is indicated by the sender through meta tags in the compound message and triggers a second communication encounter between sending and receiving process.

The first approach typically leads to many messages of small sizes (for the test example computation described above, there are of the order of $10^{3}$ messages with an average size of $\approx 1.5 \mathrm{kB}$ per time step), but the message exchange is initiated as soon as the send data is ready, which in principle allows for 
concurrency between computation and communication.

With the second method, messages are much larger (we chose $100 \mathrm{kB}$ for the compound message size), but they are exchanged only after a level update is complete. Message collection is performed into sliced buffers of fixed slice size which grow on demand and are reset after the communication is completed. At that point, the buffers are not de-allocated but get reused in the next data exchange in order to avoid frequent allocation-deallocation sequences. A complication here is that the number of necessary slices within a communication cycle is practically unpredictable a priori, as it depends on the order in which the smaller inter-block data is serialized due to padding effects. One way to go here would be to compute the needed total message size for every senderreceiver pair in advance and negotiate the resulting MPI message size after each regridding operation. This, however had to be done for every type of data exchange separately (boundary copy, coarse-fine interpolation, fine-coarse update, etc.) and also for each boundary transfer object, as different exchanges for different physical fields might be used in more complex simulation settings. For simplicity, we used a fixed MPI compound message size and took into account that message padding leads to some communication overhead.

For a small number of processes, both approaches gave satisfactory results, the speedup-curves for the second approach on the IBM machines is given in Figure 4 for up to 32 processors. As can be seen, the scaling is still reasonable up to 32 processors (with a speedup factor of about 26), but slightly inferior to the pure multi-threaded method.

Similar to the multi-thread case, a smaller test problem was run on the Opteron 850 quad with the LAM MPI implementation. This resulted in basically the same numbers as the multi-threaded case (Fig. 5). In all these cases, the MPI traffic was intra-machine and has been communicated through shared memory inter-process communication by the corresponding MPI transport layer.

\subsection{Hybrid Parallelization}

The two parallelization methods described so far have been combined into a hybrid mode in the obvious way: A number of processes are started, and within each, a configurable number of threads is started up to work concurrently. As before, each thread task within a process consists on a fixed subset of grid blocks, with its own copy of the grid structure and connectivity information. Thereby, all threads work autonomously in large portions of the computation, but are synchronized before data exchange operations.

Depending on the choice of the MPI data exchange mode, the inter-process 
communication differs slightly:

1. When each inter-block data message is mapped into one MPI message, all executing threads post their corresponding messages immediately after a block is updated. After a complete level update, one thread finishes the MPI requests, and all threads dispatch the received messages concurrently. This method requires a thread-safe MPI implementation and was tested with LAM MPI and LAMPI on Linux and with the IBM implementation on AIX.

2. When collecting inter-block messages into compound MPI messages, all threads serialize concurrently into the send buffers to a specific peer process. Here, the memory allocation and housekeeping within the compound message is realized in a thread-safe way by standard mutex-locking. Data serialization itself happens non-exclusively after allocation. After a levelupdate, only one main thread performs the MPI send-receive operations and queues the received compound messages, which are de-serialized and dispatched concurrently by the other threads (or the main thread itself, if there is only one thread per process).

As with the pure MPI mode, both methods were tried on the p690 machines and the comparison resulted clearly in favor of the second way, i.e. the use of compound messages for the MPI transfer. This might be surprising in the first instance, as only one main thread is in charge of the entire MPI communication (send/receive), but it must be kept in mind that 1 . the latency is fairly low (some $\mu \mathrm{s}$ ) and 2. that the serialization/de-serialization work is shared between the threads. Also, on the receiving side, de-serialization and MPI communication can overlap from the second compound message on.

A further remark is due for the first method, i.e. concurrent MPI traffic from all threads: First of all, the performance degraded seriously here, with an increase in execution time of up to $20 \%$ in some cases. Modifying the method in a way that not only one thread performed the MPI message completion, but all threads did concurrent waits, each on those messages that target their "thread-local" blocks, gave even worse results and had to be discarded entirely. This behavior indicates that the matching of many small messages during the wait stage from within different threads can cause severe penalties, at least when done in the naive way it was implemented here (where the matching of message destination and message type was realized through the MPI message tag and no distinct MPI communicators were used). Also, even with thread-safe MPI implementations, the internals of the communication layers and possible thread synchronization and serialization is not visible to the user and depends strongly on the implementation itself.

Speedup-curves for the hybrid mode obtained on the p690 are given in Figure 6 for the previously discussed test run with 32 and 64 CPUs, respectively. Here, 


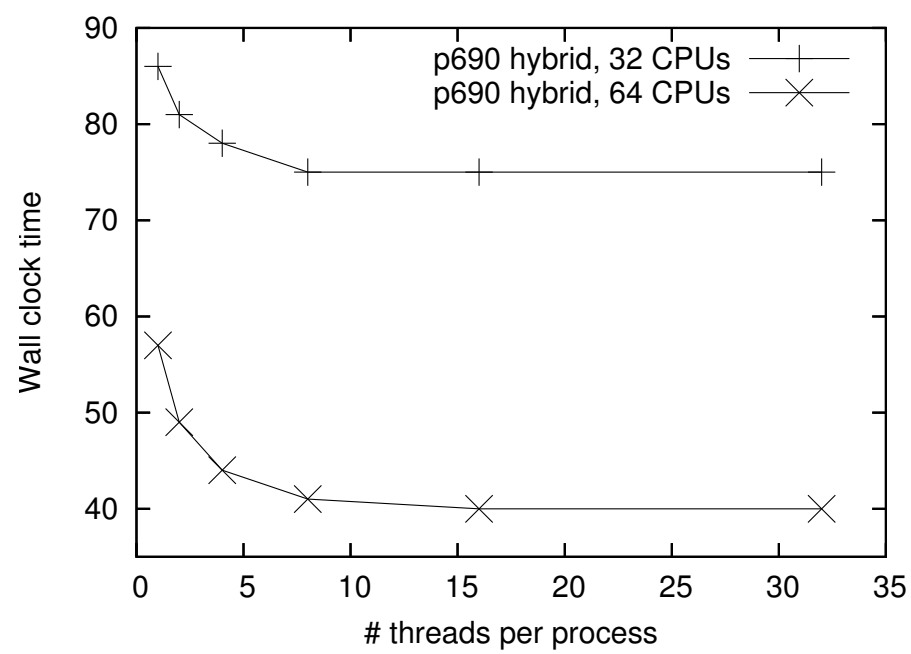

Fig. 6. Wall-clock time for test application execution in hybrid-parallelization on IBM p690 with 32 and 64 CPUs, respectively. Displayed is the execution time with 1 to 32 threads per process. The total number of threads is 32 and 64, respectively, grouped in a variable number of processes.

the total number of working threads equal the number of available CPUs, but the grouping into processes ranges from the pure MPI parallelization (1 thread per process, 32 resp. 64 processes) to the maximum of 32 threads per process (i.e. pure multi-thread mode in the case of 32 CPUs or 2 processes with 32 threads spread over 2 machines in the case of 64 CPUs). The MPI communication is based on compound messages of $100 \mathrm{kB}$ size, and the task affinity was again enforced through explicit CPU binding as described in the multi-thread section.

It is obvious that there is some performance loss with increasing numbers of processors, while the speed-up from 32 to 64 CPUs in the 32 threads case corresponds to a factor of 1.9 (76s to 40s). Comparing the most favorable runs with the pure MPI runs, we get a performance loss of $13 \%$ in the 32 CPU case (76s to $86 \mathrm{~s}$ ), and a more pronounced penalty of $42 \%$ in the $64 \mathrm{CPU}$ case $(40 \mathrm{~s}$ to $57 \mathrm{~s}$ ). These results indicate that the hybrid mode in fact can lead to a gain compared to the pure MPI parallelization once the multi-threaded execution is optimized by enforcing the tight affinity between tasks, CPUs and memory.

\section{Conclusions}

We have described the development of a new framework for mesh-adaptive computations of hyperbolic conservative systems. The project has been inspired by the attempt to create a modular basis for AMR computations which can easily be extended and specialized, and which is suited for parallel computing environments. 
Through the use of object-oriented methods, the complexity associated with AMR could be implemented successfully with limited efforts. During the iterative development cycle, we noticed that the principles of code re-use and abstraction saved us considerable efforts when changing parts of the program or correcting errors.

A regular subgridding method, now widely used [3], [18], [2], has been proven to be a straight-forward but well working approach for the mesh refinement, and the recent development of new numerical schemes for hyperbolic systems, which offer an attractive combination of simplicity, robustness and accuracy, provided a further major ingredient in the philosophy of a flexible framework.

As for parallelization, we have developed a hybrid concept of multi-threading and inter-process communication through MPI. The benchmarks on IBM p690 machines with 32 CPUs show that the hybrid concept in fact results in performance gain over a pure MPI parallelization, which, however requires a careful optimization of the multi-threaded implementation.

A key issue here is the efficient use of the CPU cache, which in the first place can be naturally obtained in AMR by the use of small grid block sizes that fit well into the cache. In addition, each thread in the current implementation creates its own effective data subspace consisting of a fixed subset of grid blocks and the block connectivity information, all allocated in the thread itself in order to achieve small CPU-memory distances in NUMA architectures. Block assignment to threads is based on the same space filling curve algorithm that determines the distribution among processes, and which thereby tends to minimize not only inter-process communication but also inter-thread memory accesses with potential cache conflicts. To finally achieve the desired gain from multi-threading, the affinity between tasks and CPUs must be enforced manually by binding the working threads to individual CPUs.

For the MPI part of the communication, it turned out that the creation of fewer messages of moderate size (1MB and below) by collecting the small inter-block messages which are addressed to the same target processor is favorable compared to mapping the typically small messages $(\approx$ one to few $\mathrm{kB})$ between blocks directly to MPI messages, despite the fact that all MPI traffic is channeled through one thread in the message collection method, Here, the (de-) serialization of compound MPI messages can occur concurrently by many threads. These results indicate that the concurrent access to the MPI layer for the completion of many small-sized messages, even with multi-thread abilities, should be used carefully with respect to the overall performance.

In the end, the hybrid concept proved to work satisfactory and resulted in floating point performances in the range of $7-10 \%$ of the theoretical peak performance on 64 processors for the described application. Naturally, there 
is still some room for further improvement, for example in connection with automatic estimates for the size of MPI compound messages. For practical use, further development of high-level interfaces for the control of task and memory affinity on high performance computers would be helpful, as the method of explicit CPU binding that was chosen here has the potential to conflict with the job dispatcher and load distribution algorithms in larger settings. One interesting initiative for IBM's platform is the VSRAC interface project (www.redbooks.ibm.com/redpapers/pdfs/redp3932.pdf), that might be extended in the near future to allow a thread-level control in addition to its current process-level control.

With respect to the performance and scaling figures given here, it should be mentioned once more that the ratio of computation to communication costs with the tailored numerical schemes discussed here is very favorable in that the communication accounts for no more than $10 \%$ of the execution time. Other schemes with more global data dependencies and more volume data communication, like for example elliptical solvers, might be more sensible to the communication overhead and therefore may require an increased effort to optimize the parallelization. These further investigations are planned for an adaptive multigrid solver which is currently under development.

\section{Acknowledgements}

We would like to thank the members of the Zentralinstitut für Angewandte Mathematik at Forschungszentrum Jülich for their assistance and advice on issues of the parallel implementation. Access to the JUMP multiprocessor computer at Forschungszentrum Jülich was made available through project HBO18. This work benefitted from support through INTAS contract 00-292 and Sonderforschungsbereich 591 of Deutsche Forschungsgemeinschaft. We thank an anonymous referee for helpful comments and suggestions on the original manuscript.

\section{References}

[1] M. J. Berger and P. Collela, Local Adaptive Mesh Refinement for Shock Hydrodynamics, J. Comp. Phys, 82, 64 (1989).

[2] M. Parashar, J.C. Brown, see http://www.caip.rutgers.edu/ parashar/DAGH.

[3] FLASH: An Adaptive Mesh Hydrodynamics Code for Modeling Astrophysical Thermonuclear Flashes, B. Fryxell, K. Olson, P. Ricker, F. X. Timmes, M. 
Zingale, D. Q. Lamb, P. MacNeice, R. Rosner, J. W. Truran, H. Tufo, Astrophys. J., 131, 273, (2000).

[4] H. Friedel, R. Grauer, C. Marliani, Adaptive mesh refinement for singular current sheets in incompressible magnetohydrodynamic flows, J. Comp. Phys., 134,190 (1997).

[5] P. D. Lax, Weak Solutions of Nonlinear Hyperbolic Equations and their Numerical Computation, Comm. Pure Appl. Math., 7 , 159, (1954).

[6] S. K. Godunov, A finite difference method for the numerical computation of discontinous solutions of the equations of fluid dynamics, Mat. Sb., 47 , 271, (1959).

[7] R. Grauer, C. Marliani, Current Sheet Formation in 3D Ideal Incompressible Magnetohydrodynamics, Physica D, 151, 175, (2001).

[8] R. Grauer, C. Marliani, K. Germaschewski, Adaptive mesh refinement for singular solutions of the incompressible Euler equations, Phys. Rev. Lett., 84, 4850, (1998).

[9] C. P. Groth, D. L. De Zeeuw, T. I. Gombosi, K. G. Powell Global 3D MHD simulation of a space weather event: CME formation, interplanetary propagation, and interaction with the magnetosphere, J. Geophys. Res., 105 , 25053, (2000).

[10] D. Hilbert, Über die stetige Abbildung einer Linie auf ein Flächenstück Math. Ann., 38 , 459, (1891).

[11] G.-S. Jiang and E. Tadmor, Nonoscillatory Central Schemes for Multidimensional Hyperbolic Conservation Laws, J. Sci. Comp., 19 , 1892 (1989).

[12] R. Keppens, M. Nool, G. Toth, J.P. Goedbloed, Adaptive Mesh Refinement for conservative systems: multi-dimensional efficiency evaluation Comp. Phys. Comm., 153 , 317 (2003).

[13] R. Keppens, G. Tóth, OpenMP parallelism for multi-dimensional grid-adaptive magnetohydrodynamic simulations, in: Proc. of 2nd International Conference on Computational Science (2002).

[14] A. Kurganov and D. Levy, A Third-Order Semidiscrete Central Scheme for Conservation Laws and Convection-Diffusion Equations, J. Sci. Comp., 22, $1461(2000)$.

[15] A. Kurganov, E. Tadmor, New High-Resolution Central Schemes for Nonlinear Conservation Laws and Convection-Diffusion Equations, J. Comp. Phys., 160, 241, (2000).

[16] P. MacNeice, K.M. Olson, C. Mobarry, R. de Fainchtein, C. Packer, PARAMESH : A parallel adaptive mesh refinement community toolkit, Comp. Phys Comm., 126 , 330 (2000). 
[17] H. Nessyahu, E. Tadmor., Non-oscillatory central differencing methods for hyperbolic conservation laws, J. Comp. Phys., 87, 408, (1990).

[18] K.G. Powell, P.L. Roe, T.J. Linde, T.I. Gombosi, D. L. De Zeeuw, A SolutionAdaptive Upwind Scheme for Ideal Magnetohydrodynamics, J. Computational Phys., 154, 284, (1999).

[19] Approximate Riemann solvers, parameter vectors, and difference schemes, P. L. Roe, J. Comp. Phys., 43, 357, (1981).

[20] SAMRAI: Development project at LLNL, see http://www.llnl.gov/CASC/SAMRAI

[21] A Survey of Several Finite Difference Methods for Systems of Nonlinear Hyperbolic Conservation Laws, G. A. Sod, J. Comp. Phys., 27 , 1, (1978).

[22] O. Steiner, M. Knölker, M. Schüssler, Dynamic Interaction of Convection with Magnetic Flux Sheets: First Results, in: Solar Surface Magetism, NATO ASI Series C, 433, ed. R. J. Rutten and C. J. Schriver, Kluwer, 1994.

[23] G. Tóth, General Code for Modeling MHD flows on Parallel Computers: Versatile Advection Code, Astrophys. Lett. Comm., 34, 245 (1996).

[24] E. F. Toro, A Weighted Average Flux Method for Hyperbolic Conservation Laws, Proc. R. Soc. London A, 423 , 401, (1989).

[25] P. Woodward, P. Colella, The Piecewise Parabolic Method (PPM) for GasDynamical Simulations, J. Comp. Phys., 54 , 115, (1984).

[26] U. Ziegler, The Effect of Rotation on the Buoyant Rise of Magnetic Flux Tubes in Accretion Disks, Astr. Astrophys., 367 , 170 (2001).

[27] S. Champeaux, T. Passot, P.-L. Sulem, Alfvén wave filamentation, J. Plasma Phys., 58 , 665 (1997).

[28] D. Laveder, T. Passot, P.L. Sulem, Transverse dynamics of dispersive Alfvén waves: I. Direct numerical evidence of filamentation, Phys. of Plasmas, 9 (1), $293(2002)$.

[29] G. W. Zumbusch, On the quality of space-filling curve induced partitions, $Z$. Angew. Math. Mech., 81 25, 2001

[30] G. W. Zumbusch, Parallel Multilevel Methods. Adaptive Mesh Refinement and Loadbalancing, Teubner, 2003. 